Vietnam Journal of Mechanics, VAST, Vol.40, No. 1 (2018), pp. 47-61

DOI:10.15625/0866-7136/10088

\title{
BUCKLING AND POSTBUCKLING OF CARBON NANOTUBE-REINFORCED COMPOSITE CYLINDRICAL PANELS SUBJECTED TO AXIAL COMPRESSION IN THERMAL ENVIRONMENTS
}

\author{
Le Thi Nhu Trang ${ }^{1}$, Hoang Van Tung ${ }^{2, *}$ \\ ${ }^{1}$ University of Transport Technology, Hanoi, Vietnam \\ ${ }^{2}$ Hanoi Architectural University, Hanoi, Vietnam \\ *E-mail: hoangtung0105@gmail.com
}

Received June 15, 2017

\begin{abstract}
This paper presents an analytical investigation on the buckling and postbuckling behavior of thin composite cylindrical panels reinforced by single walled carbon nanotubes (SWCNTs), exposed to thermal environments and subjected to uniform axial compression. Material properties of isotropic matrix phase and carbon nanotubes are assumed to be temperature dependent, and effective properties of carbon nanotube-reinforced composite (CNTRC) are functionally graded in the thickness direction and estimated by extended rule of mixture. Governing equations are based on the classical thin shell theory taking von Karman-Donnell nonlinearity and initial geometrical imperfection into consideration. Approximate solutions are assumed to satisfy simply supported boundary conditions and Galerkin procedure is applied to derive explicit expressions of buckling loads and load-deflection relation. Effects of volume fraction and distribution type of carbon nanotubes, geometrical parameters, elevated temperature and initial imperfection on the nonlinear stability of CNTRC cylindrical panels are analyzed and discussed. The novelty of the present study is that closed-form results of buckling load and nonlinear loaddeflection relation can be readily used to analyze the buckling and postbuckling behaviors of axially loaded CNTRC cylindrical panels.
\end{abstract}

Keywords: carbon nanotube-reinforced composite, cylindrical panel, buckling and postbuckling, temperature dependent properties, imperfection.

\section{INTRODUCTION}

Due to unprecedented physical and chemical properties, carbon nanotubes (CNTs) have attracted huge attention in most areas of science and engineering in recent years. No previous material has displayed the combination of superior mechanical, thermal and electrical properties as CNTs [1,2]. The single-walled carbon nanotube (SWCNT) consists of a single sheet of grapheme rolled seamlessly to form a cylinder with diameter of order of $1 \mathrm{~nm}$ and length of up to centimeters. The multi-walled carbon nanotube (MWCNT)

(C) 2018 Vietnam Academy of Science and Technology 
having diameters from 2 to $100 \mathrm{~nm}$ and lengths of tens of microns, consists of an array of such cylinders formed concentrically and separated by $0.35 \mathrm{~nm}$. These exceptional mechanical properties and extraordinary thermal and electrical properties with very large aspect ratio make CNTs ideal candidates as advanced filler materials in composites [3]. Shen [4] proposed the concept of functionally graded carbon nanotube-reinforced composite (FG-CNTRC) material properties of which are varied across the thickness direction of plate structure according to functional rules and investigated the nonlinear bending behavior of thick FG-CNTRC plates using a semi-analytical approach. Stimulated by this work of Shen, subsequent studies relating to linear buckling and postbuckling behavior of CNTRC structures have been performed. Liew and his co-workers [5-7] used a numerical approach with element-free kp-Ritz method and first order shear deformation theory (FSDT) to investigate the linear buckling of rectangular and skew FG-CNTRC plates under mechanical loads. There is no temperature effect considered in their works. Wattanasakulpong and Chaikittiratana [8] and Wang et al. [9] made use of analytical and semi-analytical solutions, respectively, to study the linear buckling of simply supported FG-CNTRC plates subjected to edge compressive loads. Based on adjacent equilibrium criterion and analytical solution, Mehrabadi et al. [10] dealt with mechanical buckling problem of CNTRC rectangular plates. Shen and Zhang [11] studied the buckling and postbuckling behavior of FG-CNTRC plates under thermal loads using a two step perturbation technique. Recently, Tung [12] presented an analytical study on the buckling and postbuckling behavior of FG-CNTRC plates under two types of thermal loads incorporating effects of elastic foundations and tangential constraints of boundary edges.

Cylindrical panels are important structures widely used in many engineering applications and the static and dynamic responses of these panels are essential problems. Basing on analytical approaches and theory of elasticity, Alibeigloo $[13,14]$ and Pourasghar and Chen [15] examined the bending behavior of FG-CNTRC cylindrical panels including the effects of piezoelectric layers [13], thermal environments [14] and elastic foundations [15]. Free vibration response of FG-CNTRC cylindrical panels has been addressed in works of Alibeigloo [16] using theory of elasticity and Kiani and his collaborator [17,18] utilizing Ritz method with different shape functions. Buckling and postbuckling behavior of FG-CNTRC cylindrical panels have been investigated in some works. Nasihatgozar et al. [19] used analytical solutions based on the thin shell theory to study the linear buckling of FG-CNTRC cylindrical panels under axial compression. Linear buckling analysis of FG-CNTRC cylindrical panels under axial compression and shear has been carried out by García et al. [20] making use of a numerical approach. Based on a higher order shear deformation shell theory and a semi-analytical approach with a two step perturbation technique which is quite cumbersome, Shen and his co-workers presented studies on the postbuckling behavior of FG-CNTRC cylindrical panels subjected to some loading conditions such as lateral pressure [21], axial compression [22] and combined loads [23] taking into account the effects of elastic foundations and thermal environments. Static buckling behavior of plates and cylindrical panels made of laminated, functionally graded and CNT composite materials have been addressed in works [24-27] using numerical methods. It is evident from the literature that analytical investigations on the buckling and postbuckling behavior of FG-CNTRC cylindrical panels are very scarce. 
This paper presents an analytical approach to investigate the buckling and postbuckling behavior of FG-CNTRC cylindrical panels exposed to thermal environments and mechanically loaded by axial compression. Material properties of isotropic matrix and carbon nanotubes are assumed to be temperature dependent and effective properties of CNTRC are estimated by extended rule of mixture. CNTs are reinforced into matrix through uniform distribution or functionally graded distribution. Approximate solutions of deflection and stress functions are assumed to satisfy simply supported boundary conditions and Galerkin method is applied to obtain explicit expressions of buckling loads and load-deflection relation. Numerical illustrations are carried out to show the effects of CNT volume fraction and distribution type, geometrical parameters, elevated temperature and initial imperfection on the buckling and postbuckling behavior of FG-CNTRC cylindrical panels. The closed-form results of the present paper have practical significances and new contribution in comparison with aforementioned investigations [20,22] relating to buckling and postbuckling behaviors of axially compressed CNTRC cylindrical panels.

\section{CARBON NANOTUBE-REINFORCED COMPOSITE CYLINDRICAL PANELS}

Consider a composite cylindrical panel of axial length $a$, circumferential length $b$, radius of curvature $R$ and thickness $h$ reinforced by aligned SWCNTs ( $H$ and $b_{1}$ are depicted for sake of comparison in later section).

The panel is defined in a coordinate system $x y z$ origin of which is at the corner of the panel on the middle plane, $x, y$ are in-plane coordinates in axial and circumferential directions of the panel, respectively, and $z$ is in the direction of inward normal to the middle surface, as shown in Fig. 1. The panel is axially compressed (i.e. in $x$ direction) by pressure $P$ uniformly distributed on curved edges $x=0, a$ and is exposed to thermal environment with uniform temperature rise. Thus, the direction of compression is the same as the aligned direction of CNTs. In this study, the SWCNTs are reinforced into isotropic polymer matrix through uniform distribution referred to as UD or functionally graded distribution in which CNTs are varied in the thickness direction according to three different patterns referred to as FG-O, FG-V and FG-X. The middle plane of the cylindrical panel is CNT-rich for the FG-O type, the top surface of the panel is CNT-rich for the FG-V type and both top and bottom surfaces of the panel are CNT-rich in case of the FG-X type, as illustrated in the Fig. 1. According to micromechanical approach, the effective properties of composite materials may be determined by Mori-Tanaka scheme or the linear rule of mixture. It is known that the rule of mixture results in simplicity and convenience in application to predict the overall material properties and global responses of the structures. A good agreement between the Mori-Tanaka scheme and the linear rule of mixture for ceramic-metal functionally graded structures has been demonstrated by Librescu et al. [28]. Very recently, García et al. [20] indicated that Mori-Tanaka approach agrees well with extended rule of mixture for determining buckling loads of CNTRC cylindrical panels under axial compression. In the present study, the extended rule of mixture is used to estimate the effective Young's moduli and shear modulus of the CNTRC cylindrical 


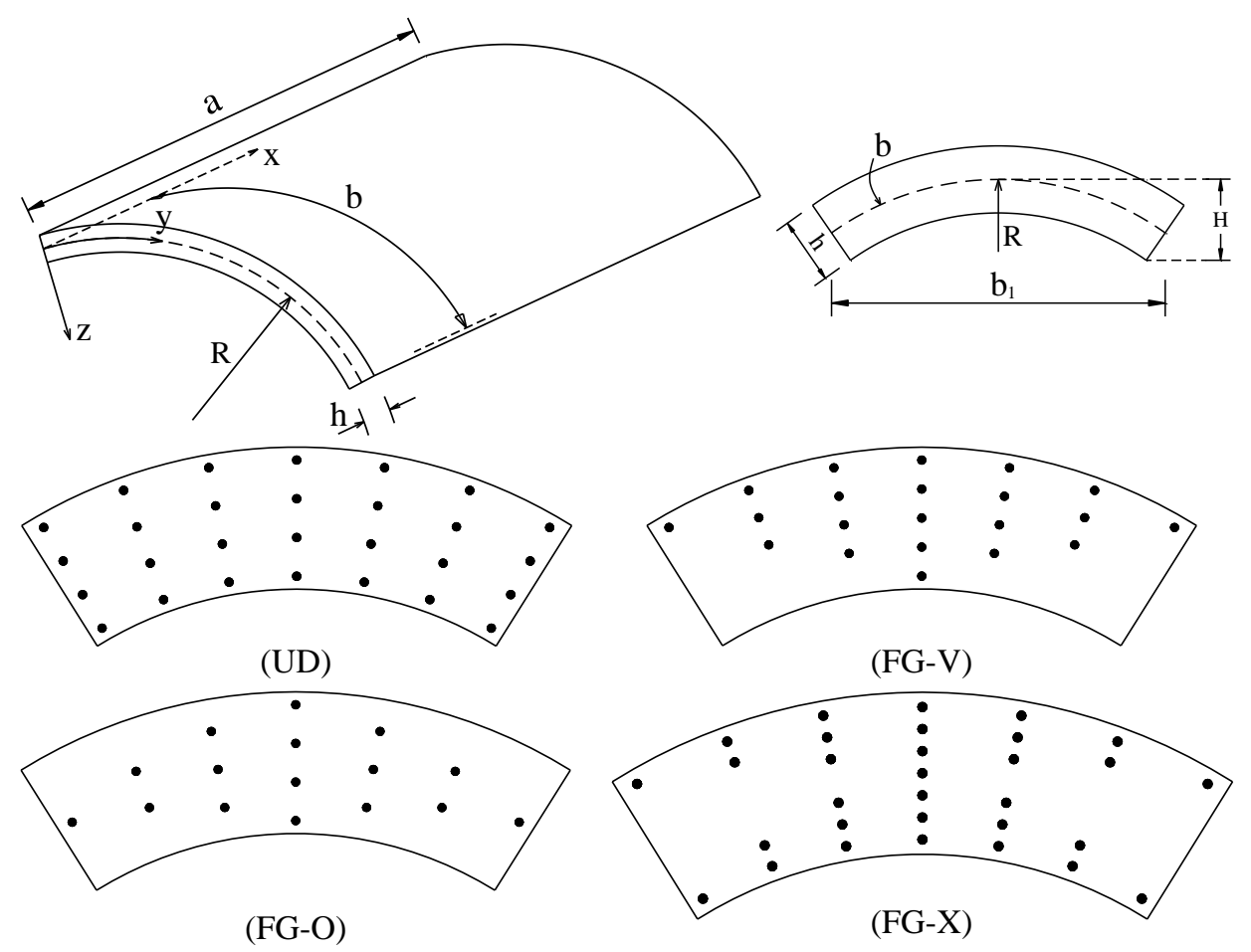

Fig. 1. Geometry, coordinate system and CNT distribution types of a cylindrical panel

panels as [4]

$$
\begin{aligned}
& E_{11}=\eta_{1} V_{C N T} E_{11}^{C N T}+V_{m} E^{m}, \\
& \frac{\eta_{2}}{E_{22}}=\frac{V_{C N T}}{E_{22}^{C N T}}+\frac{V_{m}}{E^{m}} \\
& \frac{\eta_{3}}{G_{12}}=\frac{V_{C N T}}{G_{12}^{C N T}}+\frac{V_{m}}{G^{m}},
\end{aligned}
$$

where $E_{11}^{C N T}, E_{22}^{C N T}$ and $G_{12}^{C N T}$ are the Young's moduli and shear modulus, respectively, of the carbon nanotube, and $E^{m}$ and $G^{m}$ are Young's modulus and shear modulus, respectively, of the isotropic matrix. It is noted that 1 and 2 subscripts in effective properties correspond to longitudinal direction $x$ and transverse direction $y$, respectively. The coefficients $\eta_{j}(j=1,2,3)$, known as the CNT efficiency parameters, are introduced into Eqs. (1) to account for the size-dependent material properties and will be determined later. $V_{C N T}$ and $V_{m}$ are the volume fractions of CNTs and matrix, respectively, and are related by

$$
V_{C N T}+V_{m}=1
$$


The volume fractions $V_{C N T}$ for four types of CNT distributions are assumed as [20]

$$
V_{C N T}= \begin{cases}V_{C N T}^{*}\left(1-\frac{2 z}{h}\right) V_{C N T}^{*} & (F G-V) \\ 2\left(1-\frac{2|z|}{h}\right) V_{C N T}^{*} & (F G-O) \\ 2\left(\frac{2|z|}{h}\right) V_{C N T}^{*} & (F G-X)\end{cases}
$$

where

$$
V_{C N T}^{*}=\frac{w_{C N T}}{w_{C N T}+\left(\rho_{C N T} / \rho_{m}\right)\left(1-w_{C N T}\right)}
$$

in which $w_{C N T}$ is the mass fraction of CNTs in the CNTRC panel, and $\rho_{C N T}$ and $\rho_{m}$ are the densities of the CNTs and matrix, respectively. It is noted that, in such a way, the uniformly distributed (UD) CNTRC panel, i.e. $V_{C N T}=V_{C N T}^{*}$, and the three cases of functionally graded (FG) CNTRC panel have the same value of mass fraction of the CNT. Poisson's ratio depending weakly on position can be determined by

$$
v_{12}=V_{C N T}^{*} v_{12}^{C N T}+V_{m} v^{m},
$$

where $v_{12}^{C N T}$ and $v^{m}$ are Poisson's ratios of the CNT and matrix, respectively.

The coefficients of thermal expansion of the CNTRC in the longitudinal and transverse directions have the form as $[4,11,20]$

$$
\begin{aligned}
& \alpha_{11}=V_{C N T} \alpha_{11}^{C N T}+V_{m} \alpha^{m}, \\
& \alpha_{22}=\left(1+v_{12}^{C N T}\right) V_{C N T} \alpha_{22}^{C N T}+\left(1+v^{m}\right) V_{m} \alpha^{m}-v_{12} \alpha_{11},
\end{aligned}
$$

where $\alpha_{11}^{C N T}, \alpha_{22}^{C N T}$ and $\alpha^{m}$ are thermal expansion coefficients of the CNT and isotropic matrix, respectively. Evidently, $\alpha_{11}$ and $\alpha_{22}$ are also graded in the thickness direction of the cylindrical panel.

\section{GOVERNING EQUATIONS}

Based on the classical shell theory, the strains $\varepsilon_{11}=\varepsilon_{x x}, \varepsilon_{22}=\varepsilon_{y y}, \gamma_{12}=\gamma_{x y}$ at a distance $z$ from the mid-plane are expressed as

$$
\left(\begin{array}{c}
\varepsilon_{11} \\
\varepsilon_{22} \\
\gamma_{12}
\end{array}\right)=\left(\begin{array}{c}
\varepsilon_{x 0} \\
\varepsilon_{y 0} \\
\gamma_{x y 0}
\end{array}\right)+z\left(\begin{array}{c}
k_{x} \\
k_{y} \\
2 k_{x y}
\end{array}\right)
$$

where $\varepsilon_{x 0}, \varepsilon_{y 0}$ and $\gamma_{x y 0}$ are normal and shear strains, respectively, at the middle surface of the panel, and $k_{x}, k_{y}, k_{x y}$ are changes of curvature and twist which are related to the displacement components $u, v$ and $w$ in the coordinate directions $x, y$ and $z$, respectively, as

$$
\left(\begin{array}{c}
\varepsilon_{x 0} \\
\varepsilon_{y 0} \\
\gamma_{x y 0}
\end{array}\right)=\left(\begin{array}{c}
u_{, x}+w_{, x}^{2} / 2 \\
v_{, y}-w / R+w_{, y}^{2} / 2 \\
u_{, y}+v_{, x}+w_{, x} w_{, y}
\end{array}\right), \quad\left(\begin{array}{c}
k_{x} \\
k_{y} \\
k_{x y}
\end{array}\right)=\left(\begin{array}{c}
-w_{, x x} \\
-w_{, y y} \\
-w_{, x y}
\end{array}\right),
$$


where von Karman-Donnell nonlinearity is included and subscript prime (,) represents the partial derivative.

Stress-strain relations for an anisotropic CNTRC cylindrical panel are expressed as $[5,12,17,18]$

$$
\left(\begin{array}{l}
\sigma_{11} \\
\sigma_{22} \\
\sigma_{12}
\end{array}\right)=\left(\begin{array}{ccc}
Q_{11} & Q_{12} & 0 \\
Q_{12} & Q_{22} & 0 \\
0 & 0 & Q_{66}
\end{array}\right)\left(\left\{\begin{array}{l}
\varepsilon_{11} \\
\varepsilon_{22} \\
\gamma_{12}
\end{array}\right\}-\left\{\begin{array}{c}
\alpha_{11} \\
\alpha_{22} \\
0
\end{array}\right\} \Delta T\right)
$$

where

$$
Q_{11}=\frac{E_{11}}{1-v_{12} v_{21}}, \quad Q_{22}=\frac{E_{22}}{1-v_{12} v_{21}}, \quad Q_{12}=\frac{v_{21} E_{11}}{1-v_{12} v_{21}}, \quad Q_{66}=G_{12} ，
$$

and $\Delta T$ is the temperature change with respect to a reference state. The force and moment resultants of a CNTRC cylindrical panel are expressed as

$$
\left(N_{x}, N_{y}, N_{x y}\right)=\int_{-h / 2}^{h / 2}\left(\sigma_{11}, \sigma_{22}, \sigma_{12}\right) d z, \quad\left(M_{x}, M_{y}, M_{x y}\right)=\int_{-h / 2}^{h / 2}\left(\sigma_{11}, \sigma_{22}, \sigma_{12}\right) z d z .
$$

Introduction of Eqs. (1), (3), (7) into Eqs. (9), then substituting the obtained relations into Eqs. (11) lead to the force and moment resultants in the form

$$
\left[\begin{array}{c}
N_{x} \\
N_{y} \\
N_{x y} \\
M_{x} \\
M_{y} \\
M_{x y}
\end{array}\right]=\left[\begin{array}{cccccc}
e_{11} & v_{21} e_{11} & 0 & e_{12} & v_{21} e_{12} & 0 \\
v_{12} e_{21} & e_{21} & 0 & v_{12} e_{22} & e_{22} & 0 \\
0 & 0 & e_{31} & 0 & 0 & e_{32} \\
e_{12} & v_{21} e_{12} & 0 & e_{13} & v_{21} e_{13} & 0 \\
v_{12} e_{22} & e_{22} & 0 & v_{12} e_{23} & e_{23} & 0 \\
0 & 0 & e_{32} & 0 & 0 & e_{33}
\end{array}\right]\left[\begin{array}{c}
\varepsilon_{x 0} \\
\varepsilon_{y 0} \\
\gamma_{x y 0} \\
k_{x} \\
k_{y} \\
2 k_{x y}
\end{array}\right]-\left[\begin{array}{c}
e_{11 T} \\
e_{21 T} \\
0 \\
e_{12 T} \\
e_{22 T} \\
0
\end{array}\right] \Delta T,
$$

where

$$
\begin{aligned}
& \left(e_{11}, e_{21}, e_{31}\right)=\int_{-h / 2}^{h / 2}\left(Q_{11}, Q_{22}, Q_{66}\right) d z, \quad\left(e_{12}, e_{22}, e_{32}\right)=\int_{-h / 2}^{h / 2}\left(Q_{11}, Q_{22}, Q_{66}\right) z d z, \\
& \left(e_{13}, e_{23}, e_{33}\right)=\int_{-h / 2}^{h / 2}\left(Q_{11}, Q_{22}, Q_{66}\right) z^{2} d z, \\
& e_{11 T}=\int_{-h / 2}^{h / 2} Q_{11}\left(\alpha_{11}+v_{21} \alpha_{22}\right) d z, \quad e_{21 T}=\int_{-h / 2}^{h / 2} Q_{22}\left(v_{12} \alpha_{11}+\alpha_{22}\right) d z, \\
& e_{12 T}=\int_{-h / 2}^{h / 2} Q_{11}\left(\alpha_{11}+v_{21} \alpha_{22}\right) z d z, \quad e_{22 T}=\int_{-h / 2}^{h / 2} Q_{22}\left(v_{12} \alpha_{11}+\alpha_{22}\right) z d z .
\end{aligned}
$$

The nonlinear equilibrium equations of a geometrically perfect thin cylindrical panel are expressed in the form [29]

$$
N_{x, x}+N_{x y, y}=0
$$




$$
\begin{gathered}
N_{x y, x}+N_{y, y}=0, \\
M_{x, x x}+2 M_{x y, x y}+M_{y, y y}+N_{x} w_{, x x}+2 N_{x y} w_{, x y}+N_{y} w_{, y y}+\frac{N_{y}}{R}=0 .
\end{gathered}
$$

Eqs. (14a) and (14b) are identically satisfied by a stress function $f(x, y)$ defined as

$$
N_{x}=f_{, y y}, \quad N_{y}=f_{, x x}, \quad N_{x y}=-f_{, x y} .
$$

From Eqs. (8) and (12), the nonlinear equilibrium equation (14c) of a CNTRC cylindrical panel taking into account geometrical imperfection is rewritten in the form

$$
\begin{aligned}
a_{11} w_{, x x x x} & +a_{21} w_{, y y y y}+a_{31} w_{, x x y y}+a_{41} f_{, x x y y}-f_{, y y}\left(w_{, x x}+w_{, x x}^{*}\right) \\
& +2 f_{, x y}\left(w_{, x y}+w_{, x y}^{*}\right)-f_{, x x}\left(w_{, y y}+w_{, y y}^{*}\right)-\frac{f_{, x x}}{R}=0,
\end{aligned}
$$

where $a_{11}, a_{21}, a_{31}, a_{41}$ were given in the work [12] and $w^{*}(x, y)$ is a known function representing initial geometrical imperfection. Next, strain compatibility equation of a cylindrical panel has the form [29]

$$
\varepsilon_{x 0, y y}+\varepsilon_{y 0, x x}-\gamma_{x y 0, x y}=w_{, x y}^{2}-w_{, x x} w_{, y y}-w_{, x x} / R .
$$

From Eqs. (12) and (15), the strain compatibility equation of a geometrically imperfect CNTRC cylindrical panel is rewritten in the form

$$
\begin{gathered}
a_{12} f_{, x x x x}+a_{22} f_{, x x y y}+a_{32} f_{, y y y y}+a_{42} w_{, x x x x}+a_{52} w_{, x x y y}+a_{62} w_{, y y y y} \\
-w_{, x y}^{2}+w_{, x x} w_{, y y}-2 w_{, x y} w_{, x y}^{*}+w_{, x x} w_{, y y}^{*}+w_{, y y} w_{, x x}^{*}+\frac{w_{, x x}}{R}=0,
\end{gathered}
$$

in which coefficients $a_{12}, a_{22}, a_{32}, a_{42}, a_{52}, a_{62}$ can be found in the work [12] and are omitted here for the sake of brevity.

In the present study, all edges of CNTRC cylindrical panel are assumed to be simply supported and freely movable. The associated boundary conditions are

$$
\begin{aligned}
& w=M_{x}=N_{x y}=0, N_{x}=N_{x 0} \text { at } x=0, a \\
& w=M_{y}=N_{x y}=0, N_{y}=N_{y 0} \text { at } y=0, b
\end{aligned}
$$

where $N_{x 0}=-P . h$ is prebuckling force resultant on the curved edges $x=0, a$ [29] and $N_{y 0}$ is zero-valued in the present condition of loading.

One-term approximate solution satisfying out-of-plane condition (19) is assumed as

$$
\left(w, w^{*}\right)=(W, \mu h) \sin \beta_{m} x \sin \delta_{n} y,
$$

where $W$ is the deflection amplitude, $\beta_{m}=m \pi / a, \delta_{n}=n \pi / b$ and $m, n$ are positive integers representing numbers of half waves in the $x$ and $y$ directions, respectively. Also, non-dimensional coefficient $\mu$ represents size of initial geometrical imperfection assumed to be in the form of the deflection. Next, to satisfy approximately in-plane boundary conditions (19), the stress function is assumed as

$$
f=A_{1} \cos 2 \beta_{m} x+A_{2} \cos 2 \delta_{n} y+A_{3} \sin \beta_{m} x \sin \delta_{n} y+\frac{1}{2} N_{x 0} y^{2}+\frac{1}{2} N_{y 0} x^{2},
$$

where $A_{1}, A_{2}, A_{3}$ are constant coefficients. These coefficients are determined by introducing solutions (20) and (21) into the compatibility equation (18) as 


$$
\begin{aligned}
& A_{1}=\frac{\delta_{n}^{2}}{32 a_{12} \beta_{m}^{2}} W(W+2 \mu h), \quad A_{2}=\frac{\beta_{m}^{2}}{32 a_{32} \delta_{n}^{2}} W(W+2 \mu h), \\
& A_{3}=-\frac{1}{a_{12} \beta_{m}^{4}+a_{22} \beta_{m}^{2} \delta_{n}^{2}+a_{32} \delta_{n}^{4}}\left(a_{42} \beta_{m}^{4}+a_{52} \beta_{m}^{2} \delta_{n}^{2}+a_{62} \delta_{n}^{4}-\frac{\beta_{m}^{2}}{R}\right) W .
\end{aligned}
$$

Now, the solutions (20) and (21) are substituted into the equilibrium equation (16) and applying Galerkin method for the resulting equation yield the following expression

$$
P=\frac{a_{13} B_{h}^{2}}{\pi^{2} m^{2} B_{a}^{2}} \frac{\bar{W}}{\bar{W}+\mu}+\frac{a_{23} B_{h}^{2}}{\pi^{2} m^{2} B_{a}^{2}} \bar{W}+\frac{a_{33} B_{h}^{2}}{\pi^{2} m^{2} B_{a}^{2}} \frac{\bar{W}(\bar{W}+2 \mu)}{\bar{W}+\mu}+\frac{a_{43} B_{h}^{2}}{\pi^{2} m^{2} B_{a}^{2}} \bar{W}(\bar{W}+2 \mu),
$$

where

$$
\begin{aligned}
B_{a}= & b / a, \quad B_{h}=b / h, \quad \bar{W}=W / h, \\
a_{13}= & \frac{\pi^{4}}{B_{h}^{4}}\left(m^{4} B_{a}^{4} \bar{a}_{11}+n^{4} \bar{a}_{21}+m^{2} n^{2} B_{a}^{2} \bar{a}_{31}\right) \\
& +\frac{\pi^{2} m^{2} B_{a}^{2}\left(\pi^{2} n^{2} \bar{a}_{41}+B_{h} B_{a} R_{a}\right)}{B_{h}^{4}\left(m^{4} B_{a}^{4} \bar{a}_{12}+m^{2} n^{2} B_{a}^{2} \bar{a}_{22}+n^{4} \bar{a}_{32}\right)}\left(\frac{m^{2}}{\pi^{2}} B_{a}^{3} B_{h} R_{a}-m^{4} B_{a}^{4} \bar{a}_{42}-m^{2} n^{2} B_{a}^{2} \bar{a}_{52}-n^{4} \bar{a}_{62}\right), \\
a_{23}= & \frac{32 m n \pi^{2} B_{a}^{2} \gamma_{m} \gamma_{n}}{3 B_{h}^{4}\left(m^{4} B_{a}^{4} \bar{a}_{12}+m^{2} n^{2} B_{a}^{2} \bar{a}_{22}+n^{4} \bar{a}_{32}\right)}\left(m^{4} B_{a}^{4} \bar{a}_{42}+m^{2} n^{2} B_{a}^{2} \bar{a}_{52}+n^{4} \bar{a}_{62}-\frac{m^{2}}{\pi^{2}} B_{a}^{3} B_{h} R_{a}\right), \\
a_{33}= & -\frac{2 n B_{a} R_{a}}{3 m B_{h}^{3} \bar{a}_{12}} \gamma_{m} \gamma_{n}, \quad a_{43}=\frac{\pi^{4}\left(m^{4} B_{a}^{4} \bar{a}_{12}+n^{4} \bar{a}_{32}\right)}{16 B_{h}^{4} \bar{a}_{12} \bar{a}_{32}},
\end{aligned}
$$

in which

$$
\begin{aligned}
& \left(\bar{a}_{11}, \bar{a}_{21}, \bar{a}_{31}\right)=\frac{1}{h^{3}}\left(a_{11}, a_{21}, a_{31}\right), \quad\left(\bar{a}_{41}, \bar{a}_{42}, \bar{a}_{52}, \bar{a}_{62}\right)=\frac{1}{h}\left(a_{41}, a_{42}, a_{52}, a_{62}\right), \\
& \left(\bar{a}_{12}, \bar{a}_{22}, \bar{a}_{32}\right)=h\left(a_{12}, a_{22}, a_{32}\right), \quad R_{a}=a / R, \quad \gamma_{i}=\frac{1}{2}\left[1-(-1)^{i}\right](i=m, n) .
\end{aligned}
$$

It is evident from Eq. (23) that geometrically perfect CNTRC cylindrical panels (i.e. $\mu=0$ ) exhibit a bifurcation type buckling response with the corresponding buckling compression loads are predicted as

$$
P_{b}=\frac{a_{13} B_{h}^{2}}{\pi^{2} m^{2} B_{a}^{2}}
$$

and critical buckling compression load $P_{c r}$ is the smallest value among values of $P_{b}$.

\section{RESULTS AND DISCUSSION}

Numerical results of the buckling and postbuckling analyses for CNTRC cylindrical panels subjected to axial compression load in thermal environments are presented in this section. The CNTRC panels are made of poly\{ (m-phenylenevinylene)-co-[2,5-dioctoxyp-phenylene) vinylene]\} matrix material, referred to as PmPV, reinforced by armchair (10, 10) SWCNTs. The temperature dependent properties of the PmPV material are assumed 
to be $v^{m}=0.34, \alpha^{m}=45(1+0.0005 \Delta T) \times 10^{-6} / K$ and $E^{m}=(3.51-0.0047 T) \mathrm{GPa}$ in which $T=T_{0}+\Delta T$ and $T_{0}=300 \mathrm{~K}$ (room temperature). Evidently, $\alpha^{m}=45 \times 10^{-6} / \mathrm{K}$ and $E^{m}=2.1 \mathrm{GPa}$ at room temperature. The temperature dependent material properties of the $(10,10)$ SWCNT are given by Shen and Zhang [11] and listed in Tab. 1. By matching the Young's moduli $E_{11}$ and $E_{22}$ of CNTRC obtained by the rule of mixture to those from molecular dynamics (MD) simulations reported by Han and Elliott [30], the CNT efficiency parameters $\eta_{1}$ and $\eta_{2}$ are determined and given in the work of Shen [4]. According to Shen's results [4], $\eta_{1}=0.149, \eta_{2}=0.934$ for case of $V_{C N T}^{*}=0.11 ; \eta_{1}=0.150$, $\eta_{2}=0.941$ for the case of $V_{C N T}^{*}=0.14$, and $\eta_{1}=0.149, \eta_{2}=1.381$ for the case of $V_{C N T}^{*}=0.17$. In addition, the present study assumes that $\eta_{3}: \eta_{2}=0.7: 1$ because of the lack of MD results in the work [30] for shear modulus $G_{12}$.

Table 1. Temperature dependent properties of $(10,10)$ SWCNT (tube length $=9.26 \mathrm{~nm}$, tube mean radius $=0.68 \mathrm{~nm}$, tube thickness $\left.=0.067 \mathrm{~nm}, v_{12}^{\mathrm{CNT}}=0.175\right)$ [11]

\begin{tabular}{llllll}
\hline $\begin{array}{l}\text { Temperature } \\
(\mathrm{K})\end{array}$ & $\begin{array}{l}E_{11}^{C N T} \\
(\mathrm{TPa})\end{array}$ & $\begin{array}{l}E_{22}^{C N T} \\
(\mathrm{TPa})\end{array}$ & $\begin{array}{l}G_{12}^{C N T} \\
(\mathrm{TPa})\end{array}$ & $\begin{array}{l}\alpha_{11}^{C N T} \\
\left(\times 10^{-6} / K\right)\end{array}$ & $\begin{array}{l}\alpha_{22}^{C N T} \\
\left(\times 10^{-6} / K\right)\end{array}$ \\
\hline 300 & 5.6466 & 7.0800 & 1.9445 & 3.4584 & 5.1682 \\
400 & 5.5679 & 6.9814 & 1.9703 & 4.1496 & 5.0905 \\
500 & 5.5308 & 6.9348 & 1.9643 & 4.5361 & 5.0189 \\
\hline
\end{tabular}

To validate the present approach, the buckling behavior of a CNTRC cylindrical panel subjected to uniform axial compression load is considered at room temperature. Critical buckling compression loads for CNTRC cylindrical panels are calculated by using closed-form expression (26) and listed in Tab. 2 in comparison with results given by García et al. [20] using shell finite elements. As can be seen, a good agreement is achieved in this comparison.

Table 2. Comparisons of critical buckling load intensity factor $\left(k_{\sigma}=P_{c r} / \sigma_{E}, \sigma_{E}=\left(\pi^{2} E_{m} / 12(1-\right.\right.$ $\left.\left.\left.v_{m}^{2}\right)\right)(h / b)^{2}\right)$ of perfect CNTRC cylindrical panels under axial compression $\left(b_{1}=100 \mathrm{~cm}, a=2 b_{1}\right.$, $h=b_{1} / 50, V_{C N T}^{*}=0.11, T=300 \mathrm{~K}$, see Fig. 1 )

\begin{tabular}{llllll}
\hline$H / R$ & Source & UD & FG-V & FG-O & FG-X \\
\hline 0 & García et al. [20] & 15.57 & 13.76 & 10.52 & 20.68 \\
& Present & $14.11(1,1)^{i}$ & $11.45(1,1)$ & $9.57(1,1)$ & $18.72(1,1)$ \\
\hline \multirow{2}{*}{0.45} & García et al. [20] & 121.13 & 120.76 & 99.79 & 131.73 \\
& Present & $134.92(2,2)$ & $142.62(2,3)$ & $123.21(2,2)$ & $148.56(1,2)$ \\
\hline \multirow{2}{*}{0.9} & García et al. [20] & 268.10 & 268.04 & 223.02 & 310.36 \\
& Present & $276.91(2,3)$ & $270.25(2,3)$ & $241.37(2,3)$ & $323.96(2,3)$ \\
\hline
\end{tabular}

${ }^{i}$ The number in brackets indicate the buckling mode $(m, n)$ 
The remainder of this section presents numerical results for CNTRC cylindrical panels with square planform $(a=b)$ under uniform axial compression in the thermal environments. The effects of CNT volume fraction $V_{C N T}^{*}, C N T$ distribution patterns, thermal environments and geometrical parameters on the critical buckling loads of geometrically perfect CNTRC cylindrical panels under axial compression are given in Tabs. 3 and 4 .

Table 3. Effects of CNT volume fraction and distribution type on the critical buckling loads $P_{c r}(\mathrm{MPa})$ of CNTRC cylindrical panels under axial compression in the thermal environments $[a / b=1, b / h=50, a / R=0.3,(m, n)=(1,1)]$

\begin{tabular}{llllll}
\hline$T(\mathrm{~K})$ & $V_{\text {CNT }}^{*}$ & UD & FG-V & FG-O & FG-X \\
\hline 300 & 0.11 & 37.24 & 27.96 & 21.83 & 52.72 \\
& 0.14 & 46.04 & 33.93 & 26.34 & 65.86 \\
& 0.17 & 57.40 & 42.95 & 33.55 & 81.52 \\
\hline \multirow{2}{*}{400} & 0.11 & 35.30 & 25.96 & 20.14 & 50.51 \\
& 0.14 & 43.92 & 31.80 & 24.54 & 63.40 \\
& 0.17 & 54.43 & 39.93 & 30.98 & 78.09 \\
\hline 500 & 0.11 & 33.58 & 24.12 & 18.56 & 48.63 \\
& 0.14 & 42.09 & 29.86 & 22.88 & 61.37 \\
& 0.17 & 51.80 & 37.13 & 28.58 & 75.18 \\
\hline
\end{tabular}

Table 4. Effects of geometrical parameters on the critical buckling loads $P_{c r}(\mathrm{MPa})$ of CNTRC cylindrical panels under axial compression in a thermal environment

$$
\left(a / b=1, V_{C N T}^{*}=0.14, T=400 \mathrm{~K}\right)
$$

\begin{tabular}{llllll}
\hline$a / R$ & $b / h$ & UD & FG-V & FG-O & FG-X \\
\hline 0.3 & 40 & $66.74(1,1)^{i}$ & $47.62(1,1)$ & $36.43(1,1)$ & $97.16(1,1)$ \\
& 50 & $43.92(1,1)$ & $31.80(1,1)$ & $24.54(1,1)$ & $63.40(1,1)$ \\
& 100 & $13.50(1,1)$ & $10.62(1,1)$ & $8.68(1,1)$ & $18.39(1,1)$ \\
\hline 0.5 & 40 & $72.72(1,1)$ & $54.08(1,1)$ & $42.46(1,1)$ & $103.19(1,1)$ \\
& 50 & $49.91(1,1)$ & $38.18(1,1)$ & $30.57(1,1)$ & $69.43(1,1)$ \\
& 100 & $15.82(1,2)$ & $13.25(1,2)$ & $10.72(1,2)$ & $21.03(1,2)$ \\
\hline 0.8 & 40 & $87.30(1,1)$ & $69.43(1,1)$ & $56.80(1,2)$ & $117.90(1,1)$ \\
& 50 & $59.33(1,2)$ & $48.58(1,2)$ & $38.90(1,2)$ & $80.16(1,2)$ \\
& 100 & $20.08(1,2)$ & $17.91(1,2)$ & $15.02(1,2)$ & $25.34(1,2)$ \\
\hline
\end{tabular}

${ }^{i}$ The number in brackets indicate the buckling mode $(m, n)$

Tab. 3 indicates that FG-O and FG-X types of CNT distribution give the smallest and highest values of critical buckling loads, respectively, and critical loads corresponding to uniform distribution (UD) of CNT filler are considerably higher than those corresponding to FG-O and FG-V patterns of CNT distribution. In addition, this table also demonstrates that the enhancement of CNT volume fraction $V_{C N T}^{*}$ and environment temperature $T$ 
lead to an increase and decrease, respectively, in critical buckling loads of CNTRC cylindrical panels.

Effects of geometrical ratios $a / R=(0.3,0.5,0.8)$ and $b / h=(40,50,100)$ on the critical buckling loads $P_{c r}$ of CNTRC cylindrical panels are given in Tab. 4 . It is evident from the Tab. 4 that the critical buckling loads of CNTRC cylindrical panels subjected to axial compression in a thermal environment are increased when length-to-radius $a / R$ ratio increases and remarkably decreased as side-to-thickness $b / h$ ratio is increased. Furthermore, thicker and shallower panels (i.e. smaller values of $b / h$ and $a / R$ ratios) are usually buckled at fundamental mode $(m, n)=(1,1)$, whereas the buckling response of thinner and deeper panels occurs at mode shape $(m, n)=(1,2)$. In addition, difference between critical loads corresponding to different types of CNT distribution becomes smaller as $b / h$ ratio is larger.

Effects of CNT volume fraction and distribution type, geometrical ratios, thermal environments and initial imperfection on the postbuckling behavior of CNTRC cylindrical panels under axial compression are graphically given in the following.

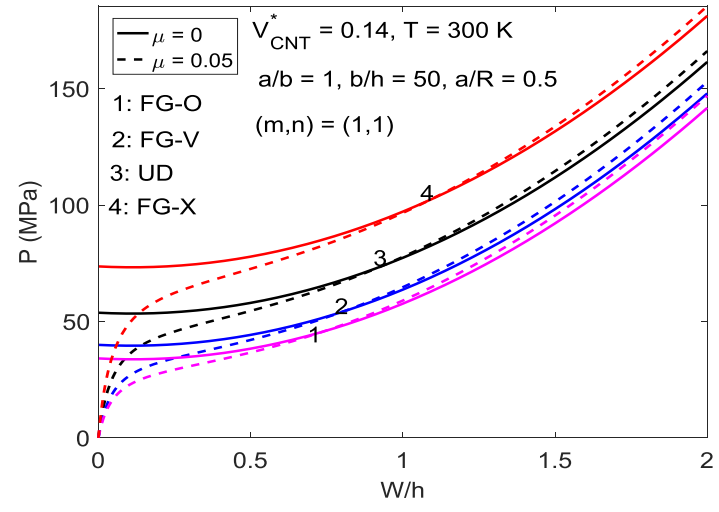

Fig. 2. Effects of CNT distribution type on the postbuckling of CNTRC cylindrical panel

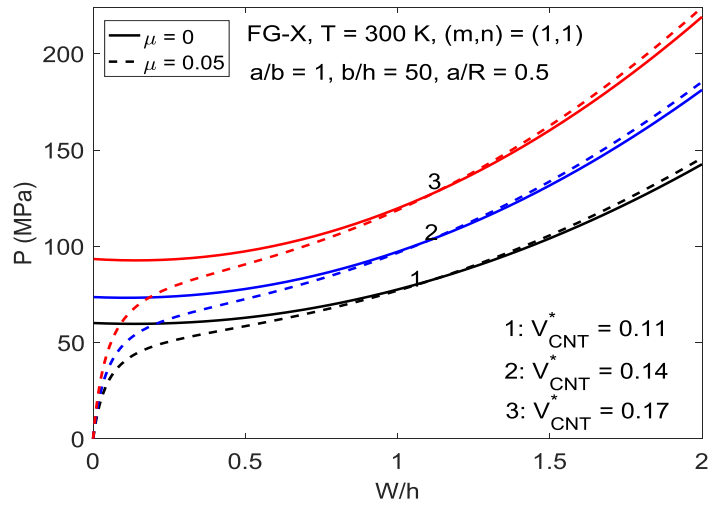

Fig. 3. Effects of CNT volume fraction on the postbuckling of CNTRC cylindrical panel

Fig. 2 gives the effects of different types of $\mathrm{CNT}$ distribution on the postbuckling of CNTRC cylindrical panels under axial compression. It is evident that the FG-X type of CNT distribution yields the highest postbuckling strength, UD type brings to better load carrying capacity than the remaining two types of functionally graded distribution, and the load-deflection curves corresponding to FG-O type of CNT distribution are the lowest. These illustrations confirm a fact that the buckling resistance and postbuckling loading capacities of CNTRC cylindrical panels are pronouncedly improved as CNTs are reinforced closer to two surfaces of cylindrical panels. In addition, postbuckling behavior of CNTRC cylindrical panels is very stable with extremely benign snap-through response. This character is an advantage of CNTRC cylindrical panels in comparison with ceramic-metal functionally graded panels [29] under axial compression because an intense snap-through response can cause fracture of cylindrical shell panels. Next, Fig. 3 shows that the volume percentage of CNT reinforcement has very sensitive influences on the postbuckling behavior of CNTRC cylindrical panels and load-deflection curves are significantly enhanced when the volume fraction $V_{C N T}^{*}$ is increased. 
Subsequently, the effects of geometrical ratios $a / R(=0.3,0.5,0.8)$ and $b / h$ $(=40,50,80)$ on the postbuckling of CNTRC cylindrical panels under axial compression are analyzed in Fig. 4. As can be observed, the buckling compression loads and postbuckling loading carrying capability of thicker and shallower CNTRC cylindrical panels (i.e. smaller values of $b / h$ and $a / R$ ratios) are remarkably higher than those of thinner and deeper panels (i.e. larger values of $b / h$ and $a / R$ ratios). In other words, the effect of the $b / h$ ratio on the buckling and postbuckling behaviors of CNTRC cylindrical panels is more impressive than that of the $a / R$ ratio. Moreover, load-deflection equilibrium paths of geometrically perfect and imperfect panels are more slowly developed when $a / R$ and $b / h$ ratios become larger.

Next, Fig. 5 examines the effects of thermal environments $(T=300 \mathrm{~K}, 400 \mathrm{~K}$ and $500 \mathrm{~K}$ ) on the postbuckling behavior of CNTRC cylindrical panels under axial compression. It is clear that the enhancement of environment temperature causes a remarked decrease in the buckling loads and load-deflection equilibrium paths of CNTRC cylindrical panels. This fact can be explained that elevated temperature has detrimental effects on the material properties of isotropic matrix and carbon nanotubes. In addition, the Figs. 2-5 also indicate that the response of axially compressed CNTRC cylindrical panels is very sensitive to initial geometrical imperfection and there is no longer bifurcation type buckling response of CNTRC cylindrical panels as imperfection size $\mu$ is not zero-valued $(\mu=0.05)$.

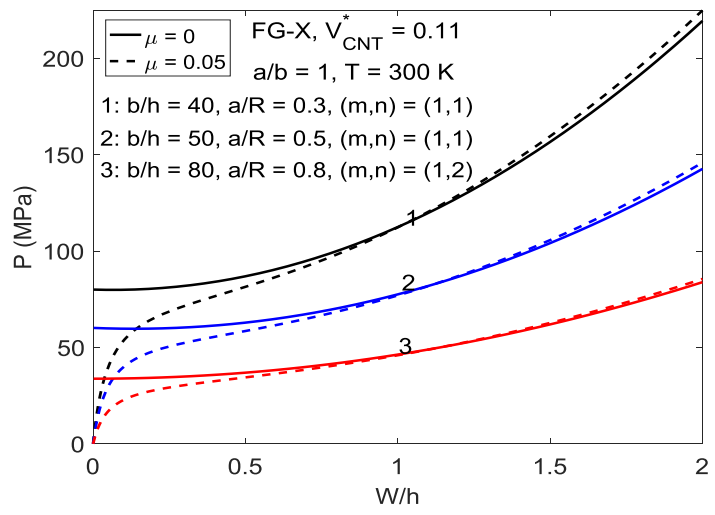

Fig. 4. Effects of geometrical ratios on the postbuckling of CNTRC cylindrical panels

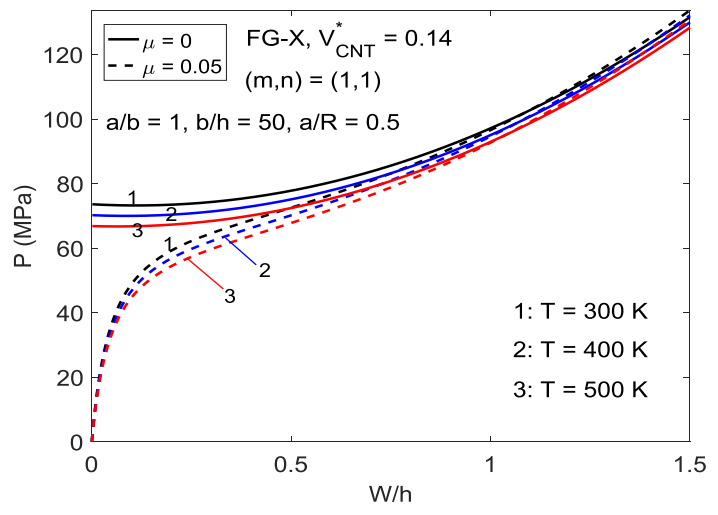

Fig. 5. Effects of thermal environments on the postbuckling of CNTRC cylindrical panels

Finally, effects of temperature on the postbuckling behavior of geometrically perfect CNTRC cylindrical panels subjected to axial compression for different volume fractions and distribution types of CNTs are analyzed in Figs. 6 and 7. Obviously, buckling loads and load-deflection curves are decreased due to the presence of elevated temperature. Furthermore, detrimental influences of temperature on the postbuckling behavior of CNTRC cylindrical panels are more pronounced for FG-X type of CNT distribution and higher volume fraction of CNTs. 


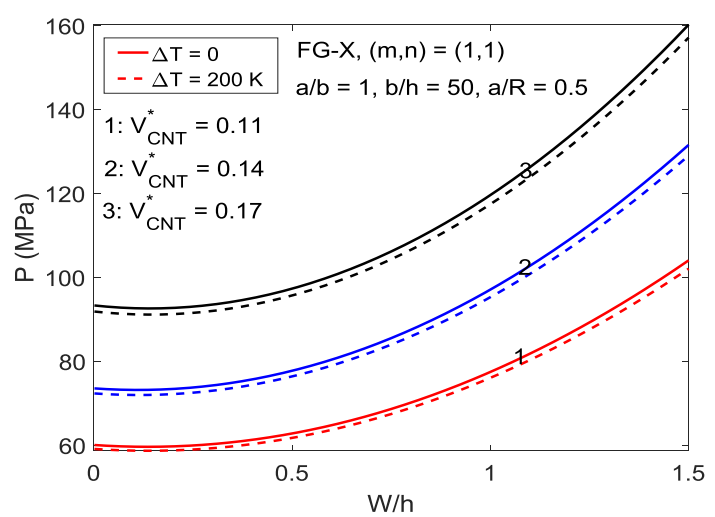

Fig. 6. Effects of temperature and CNT volume fraction on the postbuckling of perfect FGCNTRC cylindrical panels

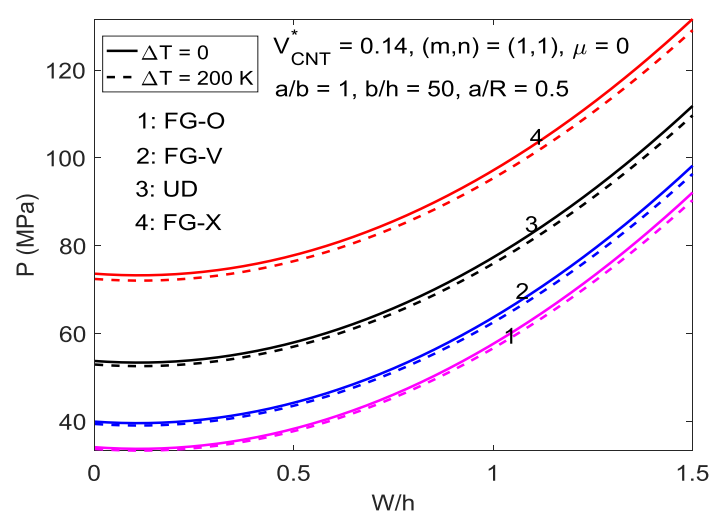

Fig. 7. Effects of temperature and CNT distribution types on the postbuckling of perfect FGCNTRC cylindrical panels

\section{CONCLUDING REMARKS}

The buckling and postbuckling behaviors of CNTRC cylindrical panels exposed to thermal environments and mechanically subjected to axial compression have been presented. The material properties of isotropic matrix and carbon nanotubes are assumed to be temperature dependent, and effective properties of CNTRC are estimated by extended rule of mixture. Formulations are based on the classical thin shell theory and explicit expressions of buckling load and load-deflection relation for simply supported CNTRC cylindrical panels are derived by using Galerkin method. The results reveal that the postbuckling behavior of axially compressed CNTRC cylindrical panels is very stable and detrimental snap-through phenomenon is extremely benign. The study also shows that the CNT volume fraction and geometrical ratios have very sensitive and significant influences on the buckling and postbuckling behaviors of CNTRC cylindrical panels. In addition, FG-X type of CNT distribution with two CNT-rich panel surfaces gives pronouncedly beneficial effects on the buckling resistance and load carrying capacities of FG-CNTRC cylindrical panels. Finally, the examination demonstrates that the enhancement of environment temperature causes a remarked decrease in the buckling compression loads and postbuckling strength of CNTRC cylindrical panels, especially for FG-X type of CNT distribution and higher volume fraction of CNTs.

\section{ACKNOWLEDGEMENT}

This research is funded by Vietnam National Foundation for Science and Technology Development (NAFOSTED) under grant number 107.02-2017.11.

\section{REFERENCES}

[1] J. N. Coleman, U. Khan, W. J. Blau, and Y. K. Gun'ko. Small but strong: A review of the mechanical properties of carbon nanotube-polymer composites. Carbon, 44, (9), (2006), pp. 16241652. doi:10.1016/j.carbon.2006.02.038. 
[2] Z. Spitalsky, D. Tasis, K. Papagelis, and C. Galiotis. Carbon nanotube-polymer composites: Chemistry, processing, mechanical and electrical properties. Progress in Polymer Science, 35, (3), (2010), pp. 357-401. doi:10.1016/j.progpolymsci.2009.09.003.

[3] E. T. Thostenson, Z. Ren, and T.-W. Chou. Advances in the science and technology of carbon nanotubes and their composites: A review. Composites Science and Technology, 61, (13), (2001), pp. 1899-1912. doi:10.1016/s0266-3538(01)00094-x.

[4] H.-S. Shen. Nonlinear bending of functionally graded carbon nanotube-reinforced composite plates in thermal environments. Composite Structures, 91, (1), (2009), pp. 9-19. doi:10.1016/j.compstruct.2009.04.026.

[5] Z. X. Lei, K. M. Liew, and J. L. Yu. Buckling analysis of functionally graded carbon nanotubereinforced composite plates using the element-free kp-Ritz method. Composite Structures, 98, (2013), pp. 160-168. doi:10.1016/j.compstruct.2012.11.006.

[6] L. W. Zhang, Z. X. Lei, and K. M. Liew. Buckling analysis of FG-CNT reinforced composite thick skew plates using an element-free approach. Composites Part B: Engineering, 75, (2015), pp. 36-46. doi:10.1016/j.compositesb.2015.01.033.

[7] Z. X. Lei, L. W. Zhang, and K. M. Liew. Buckling of FG-CNT reinforced composite thick skew plates resting on Pasternak foundations based on an element-free approach. Applied Mathematics and Computation, 266, (2015), pp. 773-791. doi:10.1016/j.amc.2015.06.002.

[8] N. Wattanasakulpong and A. Chaikittiratana. Exact solutions for static and dynamic analyses of carbon nanotube-reinforced composite plates with Pasternak elastic foundation. Applied Mathematical Modelling, 39, (18), (2015), pp. 5459-5472. doi:10.1016/j.apm.2014.12.058.

[9] M. Wang, Z.-M. Li, and P. Qiao. Semi-analytical solutions to buckling and free vibration analysis of carbon nanotube-reinforced composite thin plates. Composite Structures, 144, (2016), pp. 33-43. doi:10.1016/j.compstruct.2016.02.025.

[10] S. J. Mehrabadi, B. S. Aragh, V. Khoshkhahesh, and A. Taherpour. Mechanical buckling of nanocomposite rectangular plate reinforced by aligned and straight singlewalled carbon nanotubes. Composites Part B: Engineering, 43, (4), (2012), pp. 2031-2040. doi:10.1016/j.compositesb.2012.01.067.

[11] H.-S. Shen and C.-L. Zhang. Thermal buckling and postbuckling behavior of functionally graded carbon nanotube-reinforced composite plates. Materials \& Design, 31, (7), (2010), pp. 3403-3411. doi:10.1016/j.matdes.2010.01.048.

[12] H. V. Tung. Thermal buckling and postbuckling behavior of functionally graded carbon-nanotube-reinforced composite plates resting on elastic foundations with tangential-edge restraints. Journal of Thermal Stresses, 40, (5), (2017), pp. 641-663. doi:10.1080/01495739.2016.1254577.

[13] A. Alibeigloo. Thermoelastic analysis of functionally graded carbon nanotube reinforced composite cylindrical panel embedded in piezoelectric sensor and actuator layers. Composites Part B: Engineering, 98, (2016), pp. 225-243. doi:10.1016/j.compositesb.2016.05.010.

[14] A. Alibeigloo. Elasticity solution of functionally graded carbon nanotube-reinforced composite cylindrical panel subjected to thermo mechanical load. Composites Part B: Engineering, 87, (2016), pp. 214-226. doi:10.1016/j.compositesb.2015.09.060.

[15] A. Pourasghar and Z. Chen. Thermoelastic response of CNT reinforced cylindrical panel resting on elastic foundation using theory of elasticity. Composites Part B: Engineering, 99, (2016), pp. 436-444. doi:10.1016/j.compositesb.2016.06.028.

[16] A. Alibeigloo. Free vibration analysis of functionally graded carbon nanotubereinforced composite cylindrical panel embedded in piezoelectric layers by using 
theory of elasticity. European Journal of Mechanics-A/Solids, 44, (2014), pp. 104-115. doi:10.1016/j.euromechsol.2013.10.002.

[17] Y. Kiani. Free vibration of FG-CNT reinforced composite spherical shell panels using Gram-Schmidt shape functions. Composite Structures, 159, (2017), pp. 368-381. doi:10.1016/j.compstruct.2016.09.079.

[18] M. Mirzaei and Y. Kiani. Free vibration of functionally graded carbon nanotube reinforced composite cylindrical panels. Composite Structures, 142, (2016), pp. 45-56. doi:10.1016/j.compstruct.2015.12.071.

[19] M. Nasihatgozar, V. Daghigh, M. Eskandari, K. Nikbin, and A. Simoneau. Buckling analysis of piezoelectric cylindrical composite panels reinforced with carbon nanotubes. International Journal of Mechanical Sciences, 107, (2016), pp. 69-79. doi:10.1016/j.ijmecsci.2016.01.010.

[20] E. García-Macías, L. Rodriguez-Tembleque, R. Castro-Triguero, and A. Sáez. Buckling analysis of functionally graded carbon nanotube-reinforced curved panels under axial compression and shear. Composites Part B: Engineering, 108, (2017), pp. 243-256. doi:10.1016/j.compositesb.2016.10.002.

[21] H.-S. Shen. Postbuckling of nanotube-reinforced composite cylindrical panels resting on elastic foundations subjected to lateral pressure in thermal environments. Engineering Structures, 122, (2016), pp. 174-183. doi:10.1016/j.engstruct.2016.05.004.

[22] H.-S. Shen and Y. Xiang. Postbuckling of axially compressed nanotube-reinforced composite cylindrical panels resting on elastic foundations in thermal environments. Composites Part B: Engineering, 67, (2014), pp. 50-61. doi:10.1016/j.compositesb.2014.06.020.

[23] H.-S. Shen and Y. Xiang. Nonlinear response of nanotube-reinforced composite cylindrical panels subjected to combined loadings and resting on elastic foundations. Composite Structures, 131, (2015), pp. 939-950. doi:10.1016/j.compstruct.2015.06.042.

[24] Ö. Civalek. Buckling analysis of composite panels and shells with different material properties by discrete singular convolution (DSC) method. Composite Structures, 161, (2017), pp. 93110. doi:10.1016/j.compstruct.2016.10.077.

[25] B. P. Patel, Y. Nath, and K. K. Shukla. Thermo-elastic buckling characteristics of angleply laminated elliptical cylindrical shells. Composite Structures, 77, (1), (2007), pp. 120-124. doi:10.1016/j.compstruct.2005.06.001.

[26] Ç. Demir, K. Mercan, and Ö. Civalek. Determination of critical buckling loads of isotropic, FGM and laminated truncated conical panel. Composites Part B: Engineering, 94, (2016), pp. 110. doi:10.1016/j.compositesb.2016.03.031.

[27] A. K. Baltacıoglu, B. Akgöz, and Ö. Civalek. Nonlinear static response of laminated composite plates by discrete singular convolution method. Composite Structures, 93, (1), (2010), pp. 153-161. doi:10.1016/j.compstruct.2010.06.005.

[28] L. Librescu, S.-Y. Oh, and O. Song. Thin-walled beams made of functionally graded materials and operating in a high temperature environment: Vibration and stability. Journal of Thermal Stresses, 28, (6-7), (2005), pp. 649-712. doi:10.1080/01495730590934038.

[29] H. V. Tung. Postbuckling behavior of functionally graded cylindrical panels with tangential edge constraints and resting on elastic foundations. Composite Structures, 100, (2013), pp. 532 541. doi:10.1016/j.compstruct.2012.12.051.

[30] Y. Han and J. Elliott. Molecular dynamics simulations of the elastic properties of polymer/carbon nanotube composites. Computational Materials Science, 39, (2), (2007), pp. 315323. doi:10.1016/j.commatsci.2006.06.011. 